University of Nebraska - Lincoln

DigitalCommons@University of Nebraska - Lincoln

May 2003

\title{
Two-active electron approach to multi-electron systems in intense ultrashort laser pulses: Application to $\mathrm{Li}^{-}$
}

G. Lagmago Kamta

University of Nebraska - Lincoln, lagmago@star500.unl.edu

Anthony F. Starace

University of Nebraska-Lincoln, astarace1@unl.edu

Follow this and additional works at: https://digitalcommons.unl.edu/physicsstarace

Part of the Physics Commons

Kamta, G. Lagmago and Starace, Anthony F., "Two-active electron approach to multi-electron systems in intense ultrashort laser pulses: Application to $\mathrm{Li}^{-1}$ (2003). Anthony F. Starace Publications. 135.

https://digitalcommons.unl.edu/physicsstarace/135

This Article is brought to you for free and open access by the Research Papers in Physics and Astronomy at DigitalCommons@University of Nebraska - Lincoln. It has been accepted for inclusion in Anthony F. Starace Publications by an authorized administrator of DigitalCommons@University of Nebraska - Lincoln. 
Published in Journal of Modern Optics 50:3/4 (2003), pp. 597-613.

DOI 10.1080/09500340210150442 Copyright (C) 2003 Taylor \& Francis Ltd.

Used by permission. http://www.tandf.co.uk/journals

Submitted March 19, 2002.

\title{
Two-active electron approach to multi-electron systems in intense ultrashort laser pulses: Application to $\mathrm{Li}^{-}$
}

\author{
G. Lagmago Kamta and Anthony F. Starace \\ Department of Physics and Astronomy, University of Nebraska-Lincoln, \\ 116 Brace Laboratory, Lincoln, NE 68588-0111, USA \\ Corresponding author- G. Lagmago Kamta, email lagmago@star500.unl.edu
}

\begin{abstract}
The interaction of $\mathrm{Li}^{-}$with an ultrashort intense laser pulse is investigated by solving the time-dependent Schrödinger equation (TDSE) in the dipole approximation using a two-active electron approach. We describe the numerical solution of the TDSE and give a more detailed presentation of a technique for obtaining angular distributions for double ionization by intense ultrashort laser pulses. We show how selection rules observed in the angular distributions for double ionization by an intense laser field can be derived from the symmetry properties of the wave function. In an attempt to elucidate the mechanisms for double ionization in the multiphoton regime, we have performed numerical experiments for ionization by single cycle and double half-cycle pulses. Our preliminary results unveil the important contribution of the shake-off mechanism, in addition to the rescattering and sequential ionization mechanisms.
\end{abstract}

\section{Introduction}

The theoretical investigation of the interaction of an atomic system with an intense ultrashort linearly polarized laser pulse requires a non-perturbative, direct numerical integration of the time-dependent Schrödinger equation (TDSE). For two-electron systems, solving the TDSE is notoriously challenging, due to the high dimensionality of the problem ( 5 dimensions for a linearly polarized laser field), which makes the numerical integration a drain of computer resources. Attempts to tackle this problem have been made recently [1-6], thanks to improvements in computer speeds and memory capacities. However, all these approaches deal only with helium or $\mathrm{H}^{-}$. For multi-electron systems having more than two electrons, attempts to solve the TDSE have been essentially limited to the single active electron approximation (SAE), which assumes that all electrons are frozen in the core and only one is allowed to interact with the laser field [7].

The first step beyond the SAE is the two-active electron approximation, for which two electrons are allowed to interact with each other and with the laser field. In this paper, we give a brief description of such an approach (see [8] for more details), which is applicable to multi-electron systems having two electrons outside a core (consisting of closed shells) that interact with an intense laser field. This approach assumes that the laser field does not influence the core electrons. We summarize our results for the electron angular distributions for double ion- 
ization of $\mathrm{Li}^{-}$by an ultrashort laser pulse and give a detailed presentation of our technique for obtaining these angular distributions.

Most intense field double ionization experiments [9-12] are performed in the tunneling regime, for which classical [11], semi-classical [13] and S-matrix [14] approaches suggest that rescattering [15] is the primary mechanism for double ionization. Despite the availability of $a b$ initio theoretical approaches to solve the TDSE for two-electron systems in its full dimensionality for linearly polarized laser pulses, calculations are usually done in the multiphoton regime, for which the Keldysh adiabaticity parameter $\gamma>1$. (The Keldysh parameter is given by $\gamma=$ $\left(I_{\mathrm{p}}=2 U_{\mathrm{p}}\right)^{1 / 2}$, where $I_{\mathrm{p}}$ is the ionization potential of the target system and $U_{\mathrm{p}}$ is the ponderomotive potential.) This is due to the fact that calculations in the tunneling regime [9], for which $\gamma \ll 1$, would require huge computer resources because of the large number of photons necessary for double ionization. For these reasons, our calculations in this paper also correspond to the multiphoton regime. In an attempt to elucidate the mechanisms for double ionization in this regime, we have investigated double ionization by single cycle and double half-cycle pulses. For the single cycle pulse (SCP), the electric field reverses direction after the first half-cycle, so that an electron wavepacket created during the first half-cycle may be driven back to the core and may possibly rescatter from it. In contrast, for the double half-cycle pulse (DHP), the electric field remains in the same direction during both half-cycles, so that no rescattering can occur. Our preliminary results show evidence of entangled contributions from various mechanisms in addition to the rescattering mechanism, such as from shake-off.

This paper is organized as follows: in section 2, we give a brief description of our numerical approach to solving the TDSE (for details, see [8]), and a more detailed presentation of the technique used to obtain angular distributions for double ionization by ultrashort laser pulses. We summarize our results for the angular distributions for double ionization of $\mathrm{Li}^{-}$in section 3 . We also discuss selection rules for multiphoton double ionization in section 3, and present initial results for our numerical experiments with a SCP and a DHP, that are designed to probe double ionization mechanisms. Unless otherwise stated, atomic units (au) are used throughout this paper.

\section{Theoretical approach}

\subsection{Solution of the time-dependent Schrödinger equation}

Consider a multi-electron system having two electrons outside closed shells. In the presence of an intense laser field such a system can be treated accurately as a two-active electron system, as long as the influence of the laser field on the core electrons is negligible. The time-dependent Schrödinger equation (TDSE) describing the interaction of the system with a laser field is

$$
\mathrm{i} \frac{\partial}{\partial t} \Psi\left(\mathbf{r}_{1}, \mathbf{r}_{2}, t\right)=[H+D(t)] \Psi\left(\mathbf{r}_{1}, \mathbf{r}_{2}, t\right)
$$

where $\mathrm{H}$ denotes the atomic Hamiltonian,

$$
H=-\frac{1}{2}\left(\nabla_{1}^{2}+\nabla_{2}^{2}\right)+V\left(r_{1}\right)+V\left(r_{2}\right)+\frac{1}{r_{12}},
$$


$r_{12}$ is the interelectronic distance, and the indices 1 and 2 refer to each of the two electrons. The potential $V(r)$ describes the interaction of each electron with the core. For $\mathrm{Li}^{-}$, this potential may be written as [16]

$$
\begin{aligned}
V(r)= & -\frac{1}{r}\left[Z_{\mathrm{c}}+\left(Z_{\mathrm{n}}-Z_{\mathrm{c}}\right) \exp \left(-a_{1} r\right)+a_{2} r \exp \left(-a_{3} r\right)\right] \\
& -\frac{\alpha_{\mathrm{c}}}{2 r^{4}}\left(1-\exp \left[-\left(r / r_{\mathrm{c}}\right)^{3}\right]\right)^{2} .
\end{aligned}
$$

Here $Z_{c}=1$ is the charge of the $\mathrm{Li}^{+}$core, $Z_{n}=3$ is the nuclear charge, and $\alpha_{c}=$ 0.1894 is the polarizability of the $\mathrm{Li}^{+}$core [17]. The core potential parameters $a_{1}$, $a_{2}$, and $a_{3}$ in equation (3) are fitted such that $V(r)$ reproduces the experimentally measured energy levels of the Li atom [18]. The operator $D(t)$, which describes the interaction of the two-active electron system with the laser field, is given in the dipole approximation by either $D(t)=\mathbf{E}(t) \cdot\left(\mathbf{r}_{1}+\mathbf{r}_{2}\right)$ for the length form or $D(t)$ $=\mathbf{A}(t) \cdot\left(\mathbf{p}_{1}+\mathbf{p}_{2}\right)$ for the velocity form. The vector potential $\mathbf{A}(t)$ and the electric field $\mathbf{E}(t)$ are given $b v$

$$
\mathbf{A}(t)=\hat{\mathbf{z}} A_{0} f(t) \sin \omega t, \quad \mathbf{E}(t)=-\frac{\partial}{\partial t} \mathbf{A}(t)
$$

where $\hat{\mathbf{z}}$ is the unit vector along the linear polarization axis of the field, $f(t)$ is the pulse envelope, which (unless stated otherwise) is assumed throughout this work to have a squared cosine form, and $\omega$ is the laser frequency.

To solve the TDSE (1), we expand the wave function in a spherical "box" $[8,19]$ :

$$
\Psi\left(\mathbf{r}_{1}, \mathbf{r}_{2}, t\right)=\sum_{L, M} \sum_{\ell_{1}, \ell_{2}} \sum_{n_{1}, n_{2}} \psi_{n_{1} n_{2}}^{\ell_{1} \ell_{2} L M}(t) \mathcal{A} \frac{R_{n_{1}, \ell_{1}}\left(r_{1}\right)}{r_{1}} \frac{R_{n_{2}, \ell_{2}}\left(r_{2}\right)}{r_{2}} \mathcal{Y}_{l_{1}, l_{2}}^{L, M}\left(\Omega_{1}, \Omega_{2}\right),
$$

where $\psi_{\mathrm{n}_{1} \mathrm{n}_{2}}^{\ell_{1} \ell_{2} \mathrm{LM}}(t)$ denotes the time-dependent expansion coefficients. The antisymmetrization operator is defined by

$$
\mathcal{A}=\frac{1+\varepsilon P_{12}}{2^{1 / 2}}
$$

where $\varepsilon=+1$ (resp. -1 ) for singlet (resp. triplet) states, and where the operator $P_{12}$ simultaneously exchanges the parameters $\left(n_{1}, \ell_{1}\right)$ and $\left(n_{2}, \ell_{2}\right)$ in order to account for the indistinguishability of the two electrons. Therefore, $\mathcal{A}$ projects onto either singlet or triplet states, so as to ensure the symmetry or antisymmetry of the spatial wave function (5) in accordance with the Pauli exclusion principle. The individual angular momenta $\ell_{1}$ and $\ell_{2}$ of the two electrons are coupled in the L-S scheme via bipolar spherical harmonics

$$
\mathcal{Y}_{l_{1}, l_{2}}^{L, M}\left(\Omega_{1}, \Omega_{2}\right)=\sum_{m_{1} m_{2}} C_{\ell_{1} m_{1} \ell_{2} m_{2}}^{L M} Y_{\ell_{2}, m_{2}}\left(\Omega_{1}\right) Y_{\ell_{2}, m_{2}}\left(\Omega_{2}\right),
$$

where $C_{\ell_{1} \ell_{1} m_{2}}^{L M}$ denotes a Clebsch-Gordan coefficient. The radial functions $R_{n, \ell}$ are obtained by numerically solving the one-electron radial Schrödinger equation

$$
\left(-\frac{1}{2} \frac{\mathrm{d}^{2}}{\mathrm{~d} r^{2}}+\frac{\ell(\ell+1)}{2 r^{2}}+V(r)\right) R(r)=\mathcal{E} R(r)
$$

in a radial box of size $r=r_{0}$ with boundary conditions $R_{n, \ell}\left(r_{0}\right)=0$, where $n$ labels the number of nodes of $R_{n, \ell}(r)$ within $r_{0}$. Note that bound $(\mathcal{E}<0)$ and continuous 
$(\mathcal{E}>0)$ one-electron radial wave functions are included in expansion (5), so that the resulting basis set is complete (except for truncation). It follows from equation (5) that the time-dependent wave function is subjected to boundary conditions at $r=r_{0}$ that are similar to that of $R_{n, \ell}(r)$, i.e. $\Psi\left(r_{1}, r_{2}=r_{0,}, t\right)=\Psi\left(r_{1}=r_{0}, r_{2}, t\right)$ $=0$. In order to minimize reflections of the probability flux that may occur at the boundaries of the box during time propagation, its size, the laser intensities and the pulse durations are adjusted such that throughout the time propagation process the wave function remains negligible at the edges of the radial box.

Since we consider a linearly polarized laser field in the dipole approximation, and because the ground state of $\mathrm{Li}^{-}$is ${ }^{1} \mathrm{~S}^{\circ}$, we can set $M=0$ in the expansion (5). Also, due to limitations in memory capacities, the expansion (5) has to be truncated by introducing cut-off values for $L, \ell_{1}, \ell_{2}, n_{1}$, and $n_{2}$. In practice, $L=0,1,2$. .., $L_{\text {max }}$ and for each $L$ selected, a limited number (about 4 to 7 ) of partial waves (i.e. $\left(\ell_{1}, \ell_{2}\right)$ pairs) is included in the expansion. Finally, for each partial wave, $N_{1}$ radial functions are selected for electron 1 and $N_{2}$ for electron 2 , i.e. $n_{1}=1,2, \ldots$, $N_{1}$ and $n_{2}=1,2, \ldots, N_{2}$. Results are considered as converged when they become stable with increasing $L_{\text {max }}$ the number of partial waves, $N_{1}$ and $N_{2}$.

Using the basis expansion (5), the TDSE takes a matrix form which, after diagonalization of the atomic Hamiltonian can be rewritten in the eigenstate representation or atomic basis [8] as

$$
i \frac{\partial}{\partial t} \boldsymbol{\Phi}(t)=[\mathbf{h}+g(t) \mathbf{W}] \mathbf{\Phi}(t)
$$

where $\mathbf{h}$ is the diagonal matrix of two-electron eigenvalues and $\mathbf{W}$ represents the dipole matrix coupling various two-electron eigenstates. The scalar function $g(t)$ equals $E(t)$ for the length gauge of the dipole interaction and $-\mathrm{i} A(t)$ for the velocity gauge. $\boldsymbol{\Phi}(t)$ is the vector representation of the wave function in the eigenstate representation. In this representation, $\boldsymbol{\Phi}(t)$ represents a linear superposition of two-electron eigenstates resulting from the diagonalization of the atomic Hamiltonian, i.e.

$$
\mathbf{\Phi}(t)=\sum_{n, L} C_{n, L} \mathbf{\Phi}_{n, L}
$$

where $\boldsymbol{\Phi}_{n, L}$ is a two-electron eigenstate of energy $E_{n^{\prime}}$ and $C_{n, L}$ is its probability amplitude. Note that one can easily move back and forth between the eigenstate representation (10) of the wave function and its coordinate representation (5) by a simple matrix-vector product [8]. The TDSE (9) is solved using a Runge-Kutta method to obtain $\Phi(t)$.

\subsection{The doubly differential double ionization probability}

In the theoretical study of the interaction of atomic systems with ultrashort, intense laser pulses, there is no exact definition for the double ionization probability (DIP), owing to the difficulty of disentangling single and double ionization contributions from the time-propagated wave function. However, to obtain the double ionization probability $P$ in intense pulsed-field calculations, one usually evaluates $[8,19,20]$ the total probability found in the region $S_{3}$ in figure 1 , which also means evaluating the integral 


$$
P=\int \mathrm{d} \Omega_{1} \int \mathrm{d} \Omega_{2} \int_{r_{1}>r_{\mathrm{c}}} \mathrm{d} r_{1} \int_{r_{2}>r_{\mathrm{c}}} \mathrm{d} r_{2}\left|\Psi\left(\mathbf{r}_{1}, \mathbf{r}_{2}, T\right)\right|^{2},
$$

where $\mathbf{r}_{j} \equiv\left(r_{j^{\prime}}, \theta_{j^{\prime}}, \phi_{j}\right)(j=1,2)$ denotes the electron spherical coordinates, and $\mathrm{d} \Omega_{j}$ $\equiv \sin \theta_{j} \mathrm{~d} \theta_{j} \mathrm{~d} \phi_{j}$ is the differential solid angle. $\Psi\left(\mathbf{r}_{1}, \mathbf{r}_{2}, T\right)$ is the antisymmetrized wave function at time $T$, the end of the laser excitation. Angular integrations in equation (11) are performed over all angles (i.e. $0 \leq \theta_{j} \leq \pi, 0 \leq \phi_{j} \leq 2 \pi$ ), while radial integrations involve only configurations where electron radial coordinates are both larger than a cut-off radius $r_{c}$. The radius $r_{c}$ depends on the system under consideration, and should be chosen such that the region $S_{0}$ contains most of the probability distribution of the initial ground state. We use $r_{c}=20$ au in our calculations, which is in accordance with the radial probability distribution for the ground state of $\mathrm{Li}^{-}$[8]. The definition (11) of the DIP is not exact because, for example, residual bound states and doubly excited states may well extend into the region $S_{3}$. These spurious contributions may be small, but they are nevertheless not negligible compared to the DIP, which is usually small as well [19].

We also consider equation (11) as our definition of the DIP, but we exclude from $\Psi\left(\mathbf{r}_{1}, \mathbf{r}_{2}, T\right)$ any spurious contributions arising from populations left at $t=T$ in atomic states below the double ionization threshold (DIT). This excludes any contributions from bound and doubly excited states, as well as singly excited states below the DIT. We proceed as follows to exclude these spurious contributions. Let $\boldsymbol{\Phi}(T)$ be the solution of the TDSE (9) at time T. In the derivation of $\Psi(T)$ $\equiv \Psi\left(\mathbf{r}_{1}, \mathbf{r}_{2}, T\right)$ in the coordinate representation from $\Phi(T)$ in the eigenstate representation, we set to zero all components of $\boldsymbol{\Phi}(T)$ corresponding to atomic states below the DIT. Thus, the resulting $\Psi(T)$ is a continuum wave function describing doubly ionized continua, as well as singly ionized continua with energies above the DIT. The radial integration in equation (11), which corresponds to summing probabilities in the domain $S_{3}$ in figure 1, is used to approximately separate the doubly ionized continua from the remaining singly ionized continua.

To obtain angular distributions for double ionization, we omit the integration over angles in equation (11). The resulting quantity,

$$
\frac{\mathrm{d}^{2} P}{\mathrm{~d} \Omega_{1} \mathrm{~d} \Omega_{2}}=\int_{r_{1}>r_{\mathrm{c}}} \mathrm{d} r_{1} \int_{r_{2}>r_{\mathrm{c}}} \mathrm{d} r_{2}\left|\Psi\left(\mathbf{r}_{1}, \mathbf{r}_{2}, T\right)\right|^{2},
$$

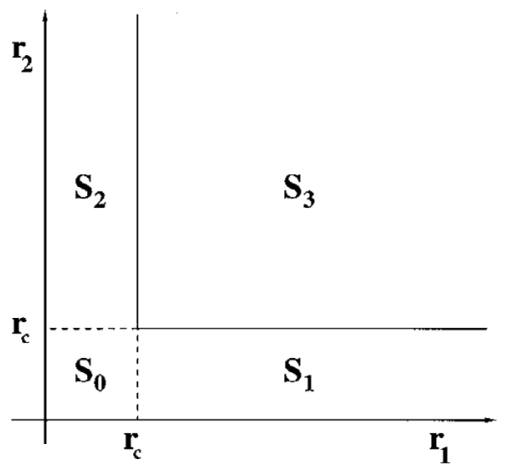

Figure 1. Spatial grid in the radial coordinates $r_{1}$ and $r_{2}$ of the two electrons. 
may be interpreted as a doubly differential, double ionization probability (DDDIP) for electron 1 to be ejected within the solid angle $\mathrm{d} \Omega_{1}$ and electron 2 within $\mathrm{d} \Omega_{2}$. The DDDIP is a function of the four spherical angles $\theta_{1}, \phi_{1}, \theta_{1}$, and $\phi_{2}$. Thus the DDDIP is in fact four-fold differential in these angles but two-fold differential in the solid angles. The DDDIP allows one to assess the role of the polarization axis $\mathbf{z}$, since $\theta_{1}$ and $\theta_{2}$ represent the angles of the two electrons with respect to this axis. Once $\Psi\left(\mathbf{r}_{1}, \mathbf{r}_{2}, T\right)$ is obtained, the DDDIP can be evaluated for any combination of the four angles mentioned above, providing thereby complete information regarding the directions of ejection of the two electrons following double ionization. Since the DDDIP is not differential in energy, it accounts for all possible energy transfers to the electrons from the laser pulse as well as for all possible energy-sharing distributions among the two electrons. The above definition of the DDDIP - as well as its possible numerical evaluation - has been made possible due to the configuration interaction structure of the basis expansion (5), which allows for an implicit separation of radial and angular integrations in equation (11). Indeed, using equation (5), the DDDIP may be expressed as

$$
\frac{\mathrm{d}^{2} P}{\mathrm{~d} \Omega_{1} \mathrm{~d} \Omega_{2}}=\frac{1}{2} \sum_{L^{\prime}, M^{\prime}} \sum_{\ell_{1}^{\prime}, \ell_{2}^{\prime}} \sum_{n_{1}^{\prime}, n_{2}^{\prime}} \psi_{n_{1}^{\prime} n_{2}^{\prime}}^{\ell_{1}^{\prime} L_{2}^{\prime} L^{\prime} M^{\prime}}(t) \sum_{L, M} \sum_{\ell_{1}, \ell_{2}} \sum_{n_{1}, n_{2}} \psi_{n_{1} n_{2}}^{\ell_{1} \ell_{2} L M}(t) \mathcal{D}\left(\Omega_{1}, \Omega_{2}\right),
$$

where

$$
\begin{aligned}
\mathcal{D}\left(\Omega_{1}, \Omega_{2}\right)= & \left\langle R_{n_{1}^{\prime}, \ell_{1}^{\prime}} \mid R_{n_{1}, \ell_{1}}\right\rangle_{r_{\mathrm{c}}}\left\langle R_{n_{2}^{\prime}, \ell_{2}^{\prime}} \mid R_{n_{2}, \ell_{2}}\right\rangle_{r_{\mathrm{c}}}\left(\mathcal{Y}_{l_{1}^{\prime}, l_{2}^{\prime}}^{L^{\prime}, M^{\prime}}\left(\Omega_{1}, \Omega_{2}\right)^{*} \mathcal{Y}_{l_{1}, l_{2}}^{L, M}\left(\Omega_{1}, \Omega_{2}\right)\right. \\
& \left.+\mathcal{Y}_{l_{2}^{\prime}, l_{1}^{\prime}}^{L^{\prime}, M^{\prime}}\left(\Omega_{1}, \Omega_{2}\right)^{*} \mathcal{Y}_{l_{2}, l_{1}}^{L, M}\left(\Omega_{1}, \Omega_{2}\right)\right) \\
& +\left\langle R_{n_{1}^{\prime}, \ell_{1}^{\prime}} \mid R_{n_{2}, \ell_{2}}\right\rangle_{r_{\mathrm{c}}}\left\langle R_{n_{2}^{\prime}, \ell_{2}^{\prime}} \mid R_{n_{1}, \ell_{1}}\right\rangle_{r_{\mathrm{c}}}\left(\mathcal{Y}_{l_{1}^{\prime}, l_{2}^{\prime}}^{L^{\prime}, M^{\prime}}\left(\Omega_{1}, \Omega_{2}\right)^{*} \mathcal{Y}_{l_{2}, l_{1}}^{L, M}\left(\Omega_{1}, \Omega_{2}\right)\right. \\
& \left.+\mathcal{Y}_{l_{2}^{\prime}, l_{1}^{\prime}}^{L^{\prime}, M^{\prime}}\left(\Omega_{1}, \Omega_{2}\right)^{*} \mathcal{Y}_{l_{1}, l_{2}}^{L, M}\left(\Omega_{1}, \Omega_{2}\right)\right),
\end{aligned}
$$

with

$$
\left\langle R_{n^{\prime}, \ell^{\prime}} \mid R_{n, \ell}\right\rangle_{r_{\mathrm{c}}} \equiv \int_{r>r_{\mathrm{c}}} R_{n^{\prime}, \ell^{\prime}}^{*}(r) R_{n, \ell}(r) \mathrm{d} r
$$

Note that the wave function coefficients $\psi_{n_{1}^{\prime} n_{2}^{\prime}}^{\ell_{1}^{\prime} \ell_{2}^{\prime} L^{\prime} M^{\prime}}(t)$ and $\psi_{n_{1} n_{2}}^{\ell_{1} \ell_{2} L M}(t)$ in equation (13) do not contain any contributions from atomic states below the DIT, which have been excluded as described above.

\section{Results}

Results presented in this paper are obtained using a spherical box of radius $r_{0}=$ $250 \mathrm{au}$, and a basis expansion with $L_{\max }=8$. The number of configurations per $L$ varies between 2700 and 3600, leading to a system of at least 20,000 ordinary differential equations to be solved. We have checked the stability of our results with respect to the box size, to $L_{\max ^{\prime}}$ to the number of partial waves, and to the number of one-electron radial functions included in the basis expansion. The binding energy obtained for $\mathrm{Li}^{-}$is $E_{\mathrm{g}}=-0.02251 \mathrm{au}(0.6125 \mathrm{eV})$, which is in good agreement with the measured value, -0.02269 au $(0.6174 \mathrm{eV})$ [21]. Figure 2 shows some energy levels of $\mathrm{Li}^{-}$obtained from our calculations. Starting from the ground state, $0.61 \mathrm{eV}$ are necessary to eject one electron from $\mathrm{Li}^{-}$, and $6.00 \mathrm{eV}$ are necessary to 


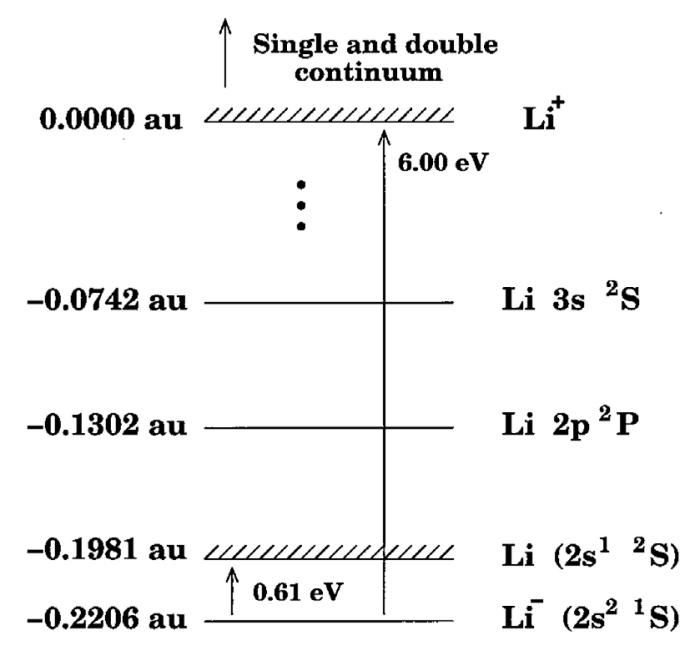

Figure 2. Energy level diagram showing the ground state of $\mathrm{Li}^{-}$, the ground state and first two excited states of $\mathrm{Li}$, and the ground state of $\mathrm{Li}^{+}$(which is taken as the zero of the energy scale). Energies corresponding to the levels are given in the left column in atomic units.

eject two electrons. Throughout this work, the initial state for the time propagation is the ground state of the system.

\subsection{The photo-detachment of $\mathrm{Li}^{-}$}

Insight into the detachment dynamics can be gained via the time evolution of the ground state population $P_{0}(t)=\left|\left\langle\Psi_{0} \mid \Psi_{L}(t)\right\rangle\right|^{2}$, where $\Psi_{0}$ is the field free ground state wave function, and $\Psi_{L}(t)$ is the solution of the TDSE at time $t$ in the length gauge. In fact, in this gauge, the projection $P_{0}(t)$ of the time-dependent wave function onto the field-free initial state $\left|\Psi_{0}\right\rangle$ can be interpreted as a probability amplitude for remaining in $\left|\Psi_{0}\right\rangle$ [22], in the velocity gauge, however, $P_{0}(t)=\mid\left\langle\Psi_{0}\right| \exp [-$ $\left.\left.\mathrm{i} \mathbf{A}(t) \cdot\left(\mathbf{r}_{1}+\mathbf{r}_{2}\right)\right] \Psi_{V}(t)\right\rangle\left.\right|^{2}$, which is numerically cumbersome to evaluate in our case. On the other hand, the mean Coulomb repulsion between the two electrons,

$$
\left\langle 1 / r_{12}\right\rangle(t)=\left\langle\Psi\left(\mathbf{r}_{1}, \mathbf{r}_{2}, t\right)\left|\frac{1}{r_{12}}\right| \Psi\left(\mathbf{r}_{1}, \mathbf{r}_{2}, t\right)\right\rangle,
$$

is gauge independent and provides insight into the dynamics of the two electrons in the laser field. Results obtained for $\left\langle 1 / r_{12}\right\rangle(t)$ in the two gauges agree very well throughout the time of interaction with the laser field [8].

Figure 3 displays the time evolution of the $\mathrm{Li}^{-}$ground state population (figure $3(\mathrm{~b}))$ and of the Coulomb repulsion $\left\langle 1 / r_{12}\right\rangle$ between the two active electrons (figure 3 (c)), for various laser peak intensities $\left(10^{10}, 5 \times 10^{10}\right.$, and $\left.10^{11} \mathrm{~W} \mathrm{~cm}^{-2}\right)$. In all cases, the laser frequency is $\omega=0.024$ au $(1898.5 \mathrm{~nm})$, and the pulse contains 4 cycles within its full width at half maximum (fwhm), which corresponds to a total of 8 laser cycles in the pulse. For reference, the electric field is plotted in figure 3(a). It appears that the ground state population oscillates with the laser field and is increasingly depleted as the laser peak intensity increases. During each halfcycle when the laser field magnitude reaches a maximum, a burst of population leaves the ground state. Note that for a peak intensity of only $5 \times 10^{10} \mathrm{~W} \mathrm{~cm}^{-2}$, al- 


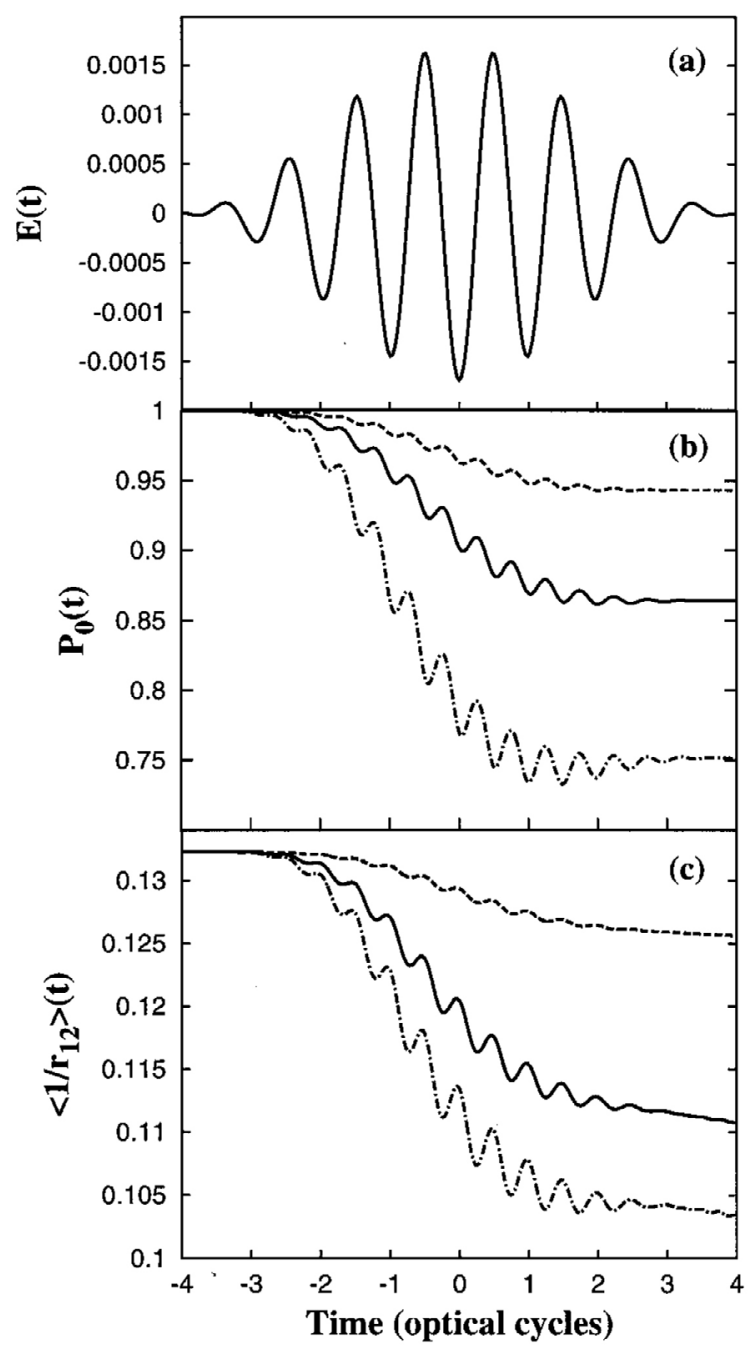

Figure 3. Time evolution of (a) the electric field $E(t)$, (b) the ground state population $P_{0}(t)$ of $\mathrm{Li}^{-}$, (c) the averaged Coulomb repulsion $\left\langle 1 / r_{12}\right\rangle$ between the two electrons. The electric field in (a) corresponds to a laser of peak intensity $I=1 \times 10^{11} \mathrm{~W} \mathrm{~cm}^{-2}$, frequency $\omega=0.024$ au $(1898.5 \mathrm{~nm})$, and pulse duration consisting of 4 cycles at fwhm. In both (b) and (c), the frequency and pulse duration are the same as in (a), while the laser peak intensities are $I=10^{10} \mathrm{~W} \mathrm{~cm}^{-2}$ (dashed line), $I=5 \times 10^{10} \mathrm{~W} \mathrm{~cm}^{-2}$ (solid line), and $I=1 \times 10^{11} \mathrm{~W} \mathrm{~cm}^{-2}$. (From [8].)

most $15 \%$ of the ground state population is depleted, indicating that even at such a fairly low intensity the behavior of $\mathrm{Li}^{-}$is already non-perturbative. Indeed, owing to the fact that $\mathrm{Li}^{-}$is a loosely bound system, non-perturbative behavior sets in at relatively low laser intensities.

Figure 3 (c) shows that the mean Coulomb repulsion between the two electrons oscillates with the laser field and decreases every half-cycle. This overall decrease is due primarily to the photo-detachment of one electron. In fact, as one electron (the "outer" electron) is photo-detached, while the other (the "inner" electron) remains bound, the probability flux associated with the detached 
electron is driven to larger distances, leading to a larger mean interelectronic distance, and the averaged Coulomb repulsion between the two electrons consequently decreases. Also, as the laser field oscillates, the Coulomb repulsion experiences a local minimum and maximum during each half-cycle. In fact, for each half-cycle, the electric field accelerates the "outer" electron to a larger distance up to a maximum, which corresponds to a local minimum (in time) in $\left\langle 1 / r_{12}\right\rangle(t)$; then the electric field changes sign and accelerates the "outer" electron towards the "inner" electron (or the core), leading to a local maximum in $\left\langle 1 / r_{12}\right\rangle(t)$. The overall decrease in the averaged Coulomb repulsion is one indication that single electron ejection dominates over double ejection [8]. As expected, it also appears that the mean Coulomb repulsion between the two electrons decreases faster as the laser peak intensity increases.

\subsection{Angular distributions for double ionization}

Angular distributions for double ionization by single photon impact in the weak field case have been subject to intensive investigation [23]. Essential features of these angular distributions are well established. We have recently shown [8] that the DDDIP described above reproduces most of these features. For example, (i) double ejection of the two electrons at large relative angles overwhelmingly dominates over two electron ejection at zero or small relative angles, due to the Coulomb repulsion between the two electrons. (ii) The DDDIP is not influenced strongly by the laser polarization axis along which the electric field drives the two electrons, thereby suggesting that this axis is largely irrelevant and that single photon double ionization is almost completely dominated by electronelectron correlations. (iii) Double ejection of the two electrons perpendicularly to the polarization axis is prevented by a selection rule that is valid regardless of the way in which the excess energy between the two electrons is shared.

For the high intensity multiphoton regime, we consider a laser pulse having a peak intensity of $I=2 \times 10^{11} \mathrm{~W} \mathrm{~cm}^{-2}$, a frequency $\omega=0.038$ au $(1199.0 \mathrm{~nm})$, and a pulse duration consisting of three laser periods within the fwhm (12 fs). The frequency is above the single ionization threshold of $\mathrm{Li}^{-}$, and six photons are necessary to reach the DIT. Three-dimensional plots of the resulting DDDIP are shown in figure 4. In order to make these plots, two of the four angles must be fixed. We have made two choices for fixing the azimuthal angles $\phi_{1}$ and $\phi_{2}:\left(\phi_{1}=\phi_{2}=0\right)$ and $\left(\phi_{1}=0, \phi_{2}=\pi\right)$, which correspond to coplanar emission of the two electrons. The laser polarization axis divides the emission plane into two half planes. For the case $\left(\phi_{1}=\phi_{2}=0\right)$, a plot of the DDDIP with respect to the polar angles $\theta_{1}\left(0 \leq \theta_{1}\right.$ $\leq \pi)$ and $\theta_{2}\left(0 \leq \theta_{2} \leq \pi\right)$ corresponds to the double ejection of the two electrons in the same half plane. The case $\left(\phi_{1}=0, \phi_{2}=\pi\right)$ corresponds to double ejection of the two electrons in different half planes (i.e. electron 1 in one half plane and electron 2 in the other).

Figure 4 shows that electrons may be ejected in all directions, mostly along the directions parallel to the laser polarization axis, thereby suggesting an enhancement of the role of the polarization axis along which the field drives electrons. Indeed, the DDDIP in figure 4 shows four prominent peaks corresponding to twoelectron ejection in the four possible configurations along the $\mathbf{z}$ axis: (i) both along positive $\mathbf{z}$ (i.e. peaks located in the vicinity of $\theta_{1}=\theta_{2}=0$ in figure 4 ), (ii) both along negative $\mathbf{z}$ (i.e. peaks located in the vicinity of $\theta_{1}=\theta_{2}=\pi$ in figure 4), (iii) 

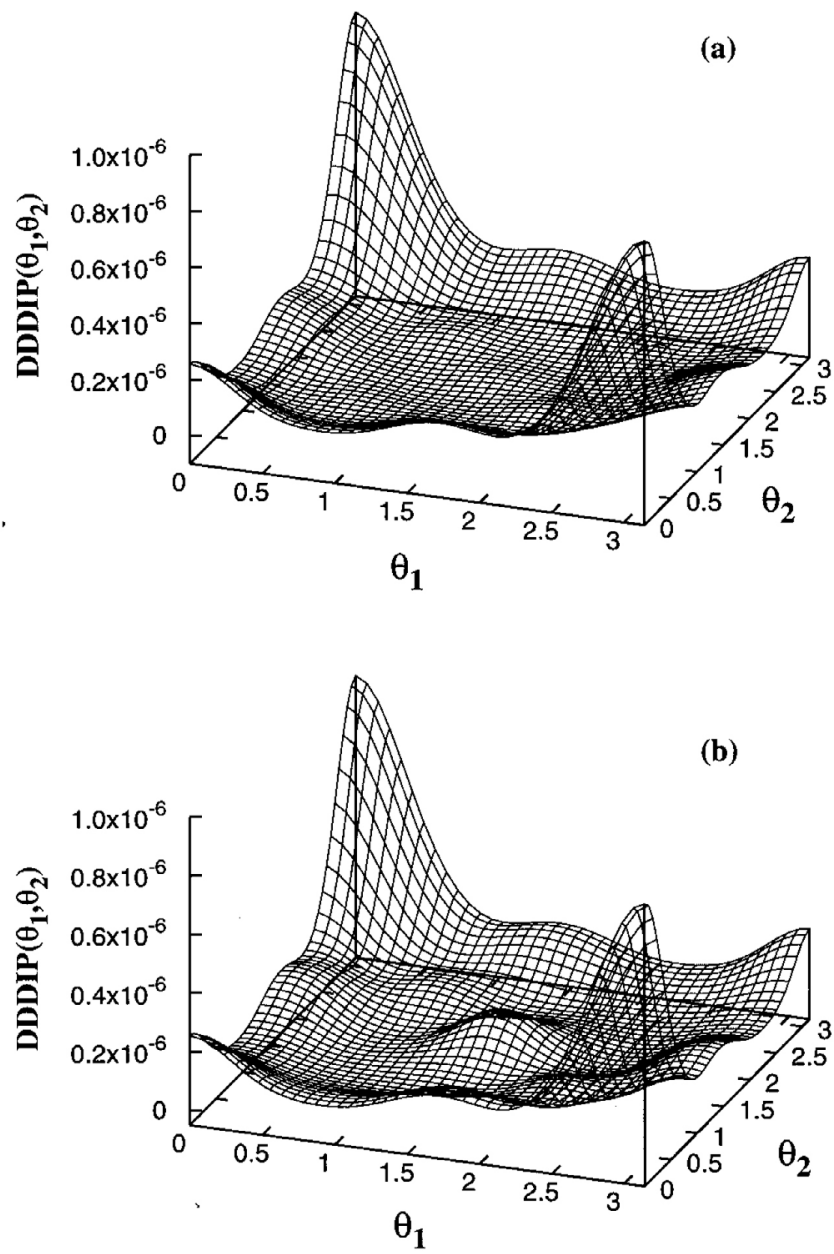

Figure 4. Doubly differential double ionization probability (DDDIP) of $\mathrm{Li}^{-}$by an intense laser pulse of peak intensity $I=2 \times 10^{11} \mathrm{~W} \mathrm{~cm}^{-2}$, frequency $\omega=0.038$ au $(1199.0 \mathrm{~nm})$ and fwhm of 12 fs. The DDDIP is plotted as a function of the angles $\theta_{1}$ and $\theta_{2}$ of the ejected electrons with respect to the polarization axis. Two coplanar cases are shown: (a) $\phi_{1}=0, \phi_{2}=0$, (b) $\phi_{1}=0, \phi_{2}=\pi$. (From [8].)

electron 1 along positive $\mathbf{z}$ and electron 2 along negative $\mathbf{z}$ (i.e. peaks located in the vicinity of $1=0,2=\mathrm{p}$ in figure 4 ), (iv) electron 2 along positive $\mathrm{z}$ and electron 1 along negative $\mathrm{z}$ (i.e. peaks located in the vicinity of $1=\mathrm{p}, 2=0$ in figure 4 ). Therefore, for an intense laser, the polarization axis along which the field drives electrons becomes highly relevant. Also, because of the intensity of the field, it is now possible for two electrons to be ejected at zero relative angle along the polarization axis. Note that the DDDIP for double electron ejection in opposite directions along the $\mathrm{z}$ axis is larger in magnitude than that corresponding to ejection in the same direction (zero relative angle) along this axis, an indication that electron-electron correlations still influence the double ejection process.

Figure 4 also shows local maxima at the angles $\theta_{1}=\theta_{2}=\pi / 2$, which correspond to two electron ejection perpendicular to the polarization axis in the same direction with zero relative angle (barely visible in figure 4 (a)) and in opposite 
directions (figure $4(\mathrm{~b})$ ). The fact that the magnitude of the DDDIP for two-electron ejection perpendicular to the laser polarization axis in opposite directions is larger than that for the case of double ejection in the same direction is another consequence of electron correlations. Therefore, in the high intensity multiphoton regime, the two electrons may be ejected perpendicular to the laser polarization axis, in contrast to the low intensity (single photon) regime, where this is prevented by selection rules. This suggests an apparent breakdown of selection rules observed for single photon double ionization.

To further investigate double ejection perpendicular to the $\mathbf{z}$ axis, we have evaluated the DDDIP using the final wave function $\Psi(T)$ from which all total angular momentum components are set to zero except one (say $L$ ), so that the corresponding $\Psi(T)$ has a defined parity $(-1)^{L}$. We have found that the DDDIP for odd L components has a node at angles $\theta_{2}=\pi / 2$ and $\theta_{2}=3 \pi / 2$, which means that there is no ejection of both electrons in the same direction $\left(\theta_{2}=\pi / 2\right)$ and in opposite directions $\left(\theta_{2}=3 \pi / 2\right)$ perpendicular to the $z$ axis. On the other hand, the DDDIP obtained from even $L$ components of $\Psi(T)$ exhibits a local maximum at angles $\theta_{2}$ $=\pi / 2$ and $\theta_{2}=3 \pi / 2$, which suggests two electron ejection perpendicular to the $z$ axis. It follows that double ejection of both electrons perpendicular to the polarization axis occurs only for even angular momentum components of the wave function. Since the wave function $\Psi(T)$ is a superposition of odd and even components, the final DDDIP contains contributions from both odd and even components, as well as from interferences between these two components. A net local maximum in the DDDIP in the direction perpendicular to the polarization axis occurs when these three contributions interfere so that they generate such a maximum.

The fact that angular distributions for odd L's vanish for double ejection of both electrons perpendicular to the polarization axis is a consequence of the following more general theorem. Let $\Psi\left(\mathbf{r}_{1}, \mathbf{r}_{2}\right)$ be a two-electron wave function describing a system that is symmetric about a fixed axis z. If $\Psi\left(\mathbf{r}_{1}, \mathbf{r}_{2}\right)$ has odd parity, then $\Psi\left(\mathbf{r}_{1}, \mathbf{r}_{2}\right)$ vanishes if both $\mathbf{r}_{1}$ and $\mathbf{r}_{2}$ are perpendicular to the $z$ axis. To prove this result, note that if $\Psi\left(\mathbf{r}_{1}, \mathbf{r}_{2}\right)$ has definite parity, then

$$
\Pi \Psi\left(\mathbf{r}_{1}, \mathbf{r}_{2}\right) \equiv \Psi\left(-\mathbf{r}_{1},-\mathbf{r}_{2}\right)= \begin{cases}+\Psi\left(\mathbf{r}_{1}, \mathbf{r}_{2}\right) & \text { for even parity, } \\ -\Psi\left(\mathbf{r}_{1}, \mathbf{r}_{2}\right) & \text { for odd parity, }\end{cases}
$$

where $\Pi$ is the parity operator, which is by definition a reflection through the origin of the position coordinates of the two electrons. It is easy to see that reflection of the electron position $\mathbf{r} \equiv(x, y, z)$ through the origin (i.e. the transformation $(x, y, z) \rightarrow(-x,-y,-z))$ is equivalent to a mirror reflection in the $x-y$ plane (i.e. the transformation $(x, y, z) \rightarrow(x, y,-z))$ followed by a rotation of the particle's position by $180^{\circ}$ about the $z$ axis (i.e. the transformation $(x, y, z) \rightarrow(-x,-y, z)$ ). Therefore, if we denote by $R_{z}$ the rotation of the two electron coordinates by an angle $180^{\circ}$ about the $z$ axis, and by $M_{x y}$ the mirror reflection of the two electron coordinates about the $x-y$ plane, then $\Pi=M_{x y} R_{z}$. If $\Psi\left(\mathbf{r}_{1}, \mathbf{r}_{2}\right)$ is symmetric with respect to the $z$ axis, then $R_{z} \Psi\left(\mathbf{r}_{1}, \mathbf{r}_{2}\right)=\Psi\left(\mathbf{r}_{1}, \mathbf{r}_{2}\right)$. If in addition $\mathbf{r}_{1}$ and $\mathbf{r}_{2}$ are both perpendicular to the $z$ axis, then $M_{x y} \Psi\left(\mathbf{r}_{1}, \mathbf{r}_{2}\right)=\Psi\left(\mathbf{r}_{1}, \mathbf{r}_{2}\right)$. This leads to

$$
\Pi \Psi\left(\mathbf{r}_{1}, \mathbf{r}_{2}\right)=M_{x y} R_{z} \Psi\left(\mathbf{r}_{1}, \mathbf{r}_{2}\right)=M_{x y} \Psi\left(\mathbf{r}_{1}, \mathbf{r}_{2}\right)=\Psi\left(\mathbf{r}_{1}, \mathbf{r}_{2}\right),
$$


for any parity of $\Psi\left(\mathbf{r}_{1}, \mathbf{r}_{2}\right)$. Comparing equations (17) and (18), it follows that $\Psi\left(\mathbf{r}_{1}\right.$, $\mathbf{r}_{2}$ ) vanishes identically if it has odd parity, thereby proving the theorem.

Starting from a ground state with $L=0$ in the dipole approximation, an odd $L$ channel is only populated by the absorption of an odd number of photons, whereas an even $L$ channel is only populated following absorption of an even number of photons. Therefore, we may conclude that ejection of both electrons perpendicular to the laser polarization axis is only due to an absorption of an even number of photons, whereas for double ionization following absorption of an odd number of photons, the two electrons cannot both be ejected in directions perpendicular to the polarization axis. In fact, the case of single photon double ionization discussed above follows this rule. This extends to the case of absorption of an arbitrary odd number of photons the selection rule (derived in [24] for single photon double ionization) that excludes double ejection of both electrons perpendicular to the laser polarization axis. Therefore exchange, parity, and angular momentum symmetry considerations affect the angular distributions for intense field multiphoton double ionization.

\subsection{Double half-cycle versus single cycle pulse}

Our calculations for the high intensity multiphoton case discussed above involve a pulse with laser frequency $\omega=0.038$ au and peak intensity $I=2 \times 10^{11} \mathrm{~W}$ $\mathrm{cm}^{-2}$. This laser frequency is above the single ionization threshold of $\mathrm{Li}^{-}$, which means that the tunneling probability is negligible. This contrasts with the tunneling regime for which the rescattering mechanism is recognized as the primary mechanism for non-sequential double ionization [11, 13, 14]. According to this mechanism, one electron is initially set free by tunneling, then accelerated in the laser field and later driven back to the core, where it ejects the second electron in an e-2e collision. In an attempt to probe the mechanism of intense field double ionization in our calculations, we consider two linearly polarized model "laser" pulses that differ in shape, but that have the same "frequency" and "peak intensity" mentioned above.* The first of these is defined by

$$
\mathbf{E}_{\mathrm{scp}}(t)= \begin{cases}\hat{\mathbf{z}} E_{0} \sin (\omega t) ; & 0 \leq t \leq 2 \pi / \omega \\ 0 ; & \text { otherwise }\end{cases}
$$

We refer to the pulse given by equation (19) as the single cycle pulse (SCP), and plot it in figure 5 (solid line). For this case, the electric field changes direction after the first half-cycle, which means that electron wavepackets created during the first half-cycle may be driven back to the parent ion during the second half-cycle. Second, we consider the pulse defined by $\mathbf{E}_{\mathrm{dhp}}(t)=\left|\mathbf{E}_{\mathrm{scp}}(t)\right|$, which is plotted in figure 5 (dashed line), and which we refer to as the double half-cycle pulse (DHP). For this case, the electric field direction is the same during both half-cycles, so that throughout the pulse, the system receives two kicks in the same direction. Therefore, in contrast to the DHP, the SCP allows for an enhancement of the

* The terms "laser," "frequency," and "peak intensity" are in quotes because unlike the SCP, the DHP is not a conventional laser pulse. However, we extend these terms to the DHP by analogy with the SCP case. We remove the quotes in subsequent references to these quantities. 


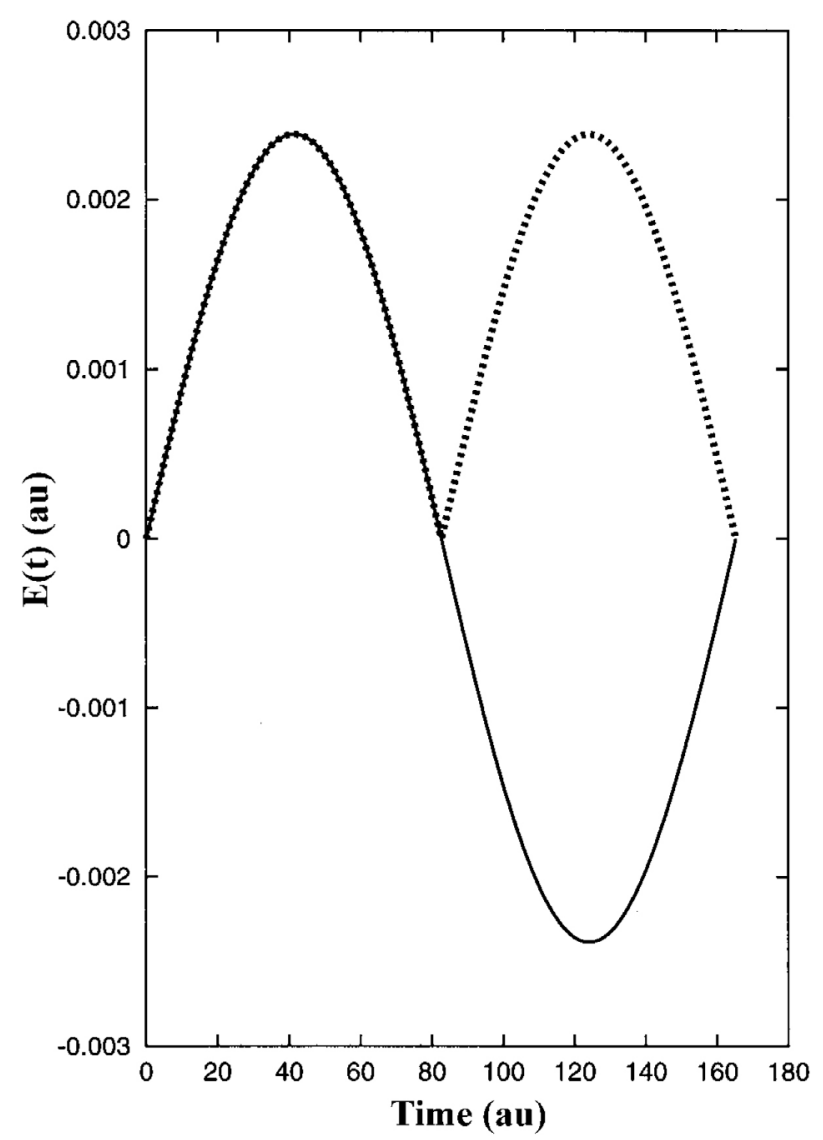

Figure 5. Time dependence of the electric field for $\omega=0.038$ au and $I=2 \times 10^{11} \mathrm{~W} \mathrm{~cm}^{-2}$. The single cycle pulse (SCP) is indicated by the solid line and the double half-cycle pulse (DHP) by the dashed line.

spatial electron-electron and electron-core correlations via the wavepacket return during the second half-cycle. Differences between the results obtained for the SCP and the DHP would clearly indicate the influence of the rescattering mechanism.

For both the SCP and the DHP, the time-dependence of the single and double ionization probabilities of $\mathrm{Li}^{-}$are shown in figures $6(\mathrm{a})$ and (b), respectively. In figure 6, only results for the second half-cycle are shown because the SCP and the DHP are identical for the first half-cycle. It appears that during the second halfcycle, the single ionization probability increases significantly for the SCP but only slightly for the DHP case. This means that the recollision that occurs for the SCP enhances single ionization. In fact, following the laser-assisted recollision that occurs for the SCP, the recolliding electrons gain significant energy to escape the nucleus, thereby enhancing single ionization.

The time dependence of double ionization plotted in figure 6(b) for both the SCP and the DHP shows the relative importance of sequential and direct double ionization. From the beginning of the second half-cycle up until it reaches its peak amplitude, both the SCP and the DHP give about the same double ioniza- 

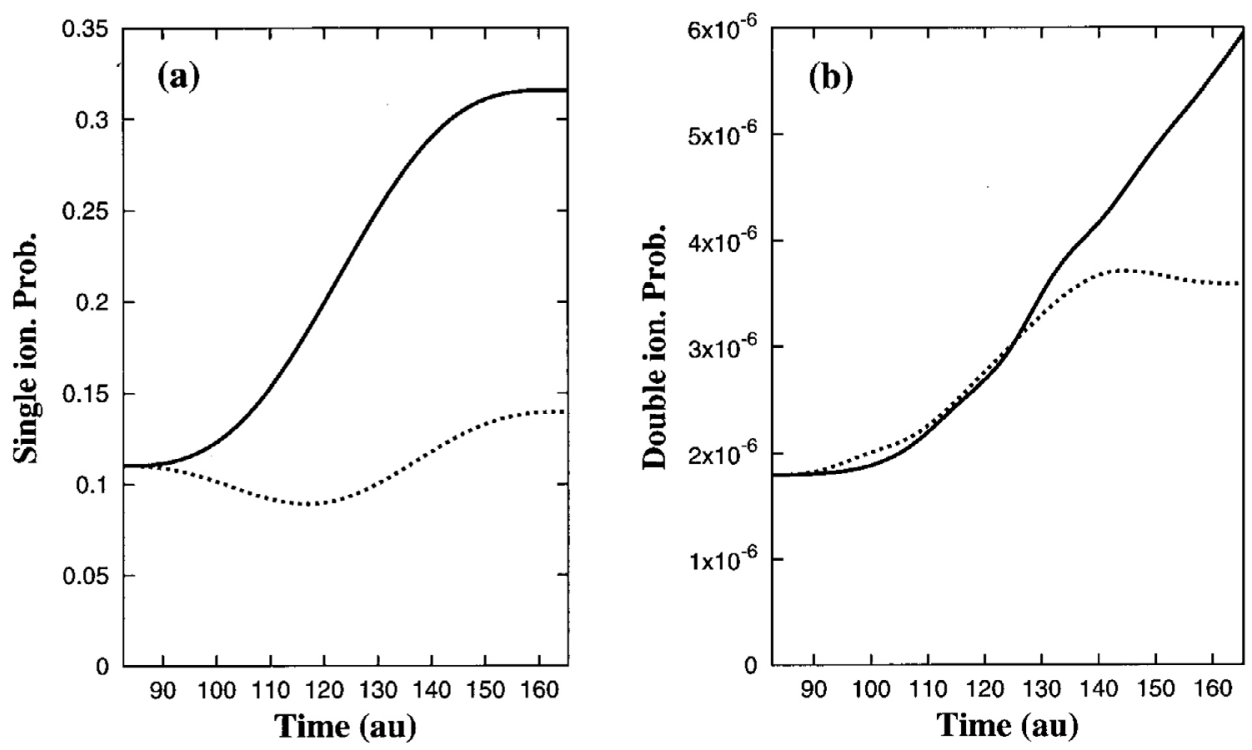

Figure 6. Single and double ionization probabilities of $\mathrm{Li}^{-}$obtained using a single cycle pulse (solid lines) and a double half-cycle pulse (dashed lines): (a) single ionization probability, (b) double ionization probability. For each case, only results corresponding to the second half-cycle are shown. The parameters used for both pulses are intensity $I=2 \times 10^{11} \mathrm{~W} \mathrm{~cm}^{-2}$, frequency $\omega=0.038$ au and pulse duration $T=2 \pi / \omega \approx 4.0 \mathrm{fs}$.

tion probability. This implies that sequential ionization (which is independent of the sign of the laser field amplitude) is predominant. For times beyond the peak amplitude of the second half-cycle, however, the SCP gives a much higher double ionization yield than the DHP. This is evidence of the role of rescattering on the double ionization yield. In fact, during this recollision, one can envision the following events. (i) The returning electron may acquire more energy in its recollision with the ion core in order to be definitely ionized, thereby leading to a larger single ionization probability for the SCP than the DHP. (ii) The returning electron may ionize the other electron in an e-2e collision process assisted by the field, leading also to an enhancement of double ionization for the SCP. The DDDIP obtained in the next section provides additional insight into another mechanism that contributes to the double ionization process besides the rescattering one described already.

The DDDIPs obtained at the end of the first half-cycle, when the field is zero, are plotted in polar coordinates in figure 7 . These results are the same for both the SCP and the half-cycle pulse, since they are identical for the first half-cycle. Also, note that selection rules described above also hold for both the DHP and the halfcycle pulse. The plots in figure 7 represent the distribution of electron 2 when electron 1 is ejected along the unit vector $\mathbf{k}_{1}$ at angles $\theta_{1}=0$ (figure $7(\mathrm{a})$ ) and $\theta_{1}=$ $\pi$ (figure $7(\mathrm{~b})$ ) with respect to the laser polarization axis. It is worth noting that during the first half-cycle, the force due to the electric field pushes the two electrons along the polarization axis in the negative direction, i.e. the field tries to eject the two electrons in the direction corresponding to the polar angles $\theta_{1}=\pi$ and $\theta_{2}=\pi$ with respect to the polarization axis. 

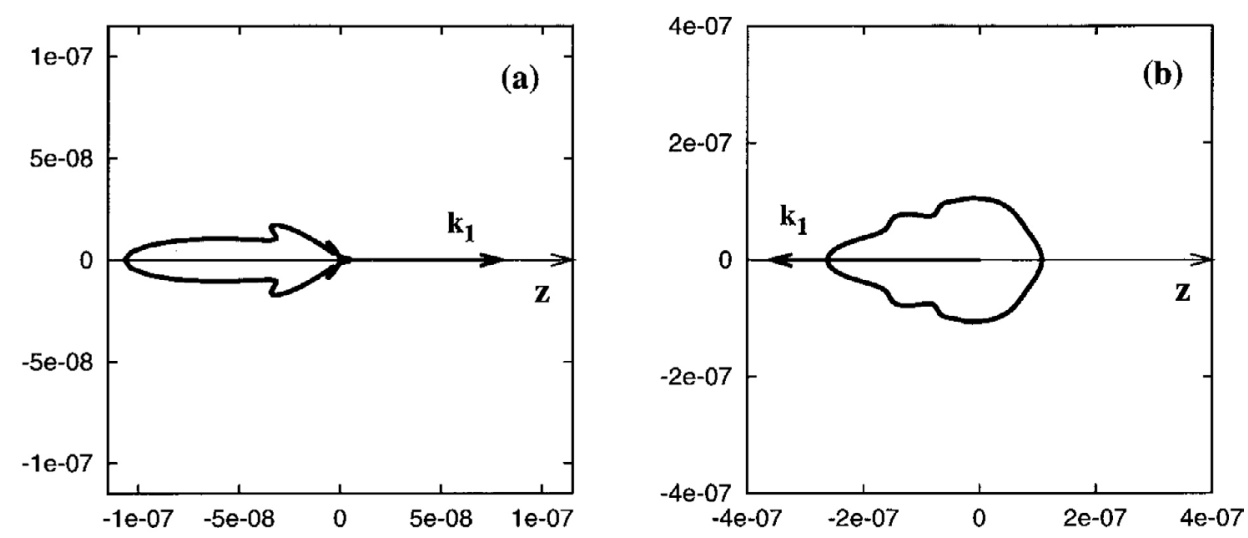

Figure 7. Plots in polar coordinates of angular distributions (coplanar) for double ionization of $\mathrm{Li}^{-}$by an intense half-cycle pulse. Results are obtained at the end of the first half-cycle for the pulses described in figure 5, and are therefore the same for the SCP and the DHP. The polar plot represents the distribution of electron 2, when electron 1 is ejected along the unit vector $\mathbf{k}_{1}$ at the angles (a) $\theta_{1}=0$ and (b) $\theta_{1}=\pi$ with respect to the polarization axis.

It appears from figure 7 (a) that if electron 1 emerges at the angle $\theta_{1}=0$, then the distribution of electron 2 is negligible for $\theta_{2}=0$. In other words, there is no ejection of both electrons along the positive direction of the polarization axis. This is expected since the field exerts no force in this direction. However, the left-hand lobe in figure $7(\mathrm{a})$ means that if electron 1 emerges at the angle $\theta_{1}=0$, electron 2 appears predominantly in the opposite direction (in the direction of the field force). This means that one electron is ejected in the direction of the field force and the other in the opposite direction. A similar configuration is also illustrated by the right-hand lobe of the plot in figure $7(b)$, which corresponds to electron 1 ejected in the direction of the field force $\left(\theta_{1}=\pi\right)$, while electron 2 has a significant probability for being ejected in the opposite direction. Electron ejection in the opposite direction of the field force is by no means due to the direct action of the field. This result contradicts a sequential uncorrelated double ionization mechanism; needless to say, it also contradicts any recollision mechanism since the field force remains in the same direction during the first half-cycle. This is a clear signature of a non-sequential double ionization that is entirely due to electron- electron correlations, in which one electron is forced to appear in the continuum in the direction opposite to the other electron or at a large relative angle with respect to the other. We believe that the mechanism involved here is shake-off [25, 26]: as the field intensity increases sharply during the first half-cycle, one electron is ejected following absorption of one or many photons, and the other electron is shaken off as it cannot adiabatically adjust to the new ionic potential. The electron that is shaken off appears predominantly in the opposite direction to the first one due to the electron repulsion.

The left-hand lobe of the plot in figure $7(\mathrm{~b})$ describes configurations where both electrons are predominantly ejected along the direction of the field force (i.e. $\theta_{1}=\pi$ and $\left.\theta_{2}=\pi\right)$ at small relative angles. This is expected for intense fields. It corresponds to sequential ionization. 


\section{Conclusions}

We have studied in the dipole approximation the interaction of $\mathrm{Li}^{-}$with an ultrashort intense laser field, linearly polarized along the $z$ axis, via a direct numerical solution of the TDSE. In all cases, angular distributions for double ionization obtained for the multiphoton regime exhibit the influence of electron- electron correlations. In the case of intense fields, both electrons may be ejected perpendicularly to the laser polarization axis; this does not occur for the single photon, weak field case. Moreover, we have shown that very general symmetry and angular momentum selection rules for a two-electron wave function having initially $L$ $=0$ permits this perpendicular ejection only for the case in which an even number of photons is absorbed.

Our numerical experiments on single and double ionization by a single cycle pulse (SCP) and by a double half-cycle pulse (DHP) show that single and double ionization are larger for the SCP than for the DHP. Since rescattering only occurs for the SCP case, this result suggests that the rescattering mechanism enhances both single and double ionization. On the other hand, angular distributions for double ionization by a half-cycle pulse, for which rescattering does not apply, show the existence of a significant shake-off contribution to double ionization, in which one electron is ejected in the direction opposite to that of the laser field force direction.

\section{Acknowledgments}

This work was supported by the U.S. Department of Energy, Office of Basic Energy Sciences, Division of Chemical Sciences, under Grant No. DE-FG03-96ER14646.

\section{References}

[1] Parker, J., Taylor, K. T., Clark, C. W., and Blodget-Ford, S., 1996, J. Phys. B: At. molec. opt. Phys., 29, L33.

[2] Scrinzi, A., and Piraux, B., 1998, Phys. Rev. A, 58, 1310; Scrinzi, A., and Piraux, B., 1997, Phys. Rev. A, 56, R13.

[3] Pindzola, M. S., and Robicheaux, F., 1998, Phys. Rev. A, 57, 318.

[4] Nikolopoulos, L. A. A., and Lambropoulos, P., 1999, Phys. Rev. Lett., 82, 3771.

[5] Lagmago Kamta, G., Grosges, Th., Piraux, B., Hasbani, R., Cormier, E., and Bachau, H., 2001, J. Phys. B: At. molec. opt. Phys., 34, 857.

[6] Hasbani, R., Cormier, E., and Bachau, H., 2000, J. Phys. B: At. molec. opt. Phys., 33, 2101.

[7] Kulander, K. C., Schafer, K. J., and Krause, J. L., 1992, Adv. at. molec. opt. Phys. Suppl., 1, 247.

[8] Lagmago Kamta, G., and Starace, A. F., 2002, Phys. Rev. A, 65, 053418.

[9] Fittingho, D. N., Bolton, P. R., Chang, B. C., and Kulander, K. C., 1992, Phys. Rev. Lett., 69, 2642.

[10] Walker, B., Sheehy, B., DiMauro, L. F., Agostini, P., Schafer, K. J., and Kulander, K. C., 1994, Phys. Rev. Lett., 73, 1227.

[11] Weber, Th., Weckenbrock, M., Staudte, A., Spielberger, L., Jagutzki, O., Mergel, V., Afaneh, F., Urbasch, G., Vollmer, M., Giessen, H., and Dörner, R., 2000, Phys. Rev. Lett., 84, 443. 
[12] Moshammer, R., Feuerstein, B., Schmitt, W., Dorn, A., Schröter, C. D., Ullrich, J., Rottke, H., Trump, C., Wittmann, M., Korn, G., Homann, K., and Sandner, W., 2000, Phys. Rev. Lett., 84, 447.

[13] Bhardwaj, V. R., Aseyev, S. A., Mehendale, M., Yudin, G. L., Villeneuve, D. M., Rayner, D. M., Ivanov, M. Yu., and Corkum, P. B., 2001, Phys. Rev. Lett., 86, 3552.

[14] Becker, A., and Faisal, F. H. M., 1999, Phys. Rev. A, 59, R1742; 2000, Phys. Rev. Lett., 84, 3546.

[15] Corkum, P. B., 1993, Phys. Rev. Lett., 71, 1994; Schafer, K. J., Yang, B., DiMauro, L. F., and Kulander, K. C., 1993, Phys. Rev. Lett., 70, 1599.

[16] Robicheaux, F., and Greene, C. H., 1992, Phys. Rev. A, 46, 3821.

[17] Johnson, W. R., Kolb, D., and Huang, K. N., 1983, At. Data Nucl. Data Tables, 28, 333.

[18] Moore, C. E., 1971, Atomic Energy Levels, National Bureau of Standards (US) No. NSRDS-NBS35 (Washington, DC: US GPO).

[19] Lagmago Kamta, G., and Starace, A. F., 2001, Phys. Rev. Lett., 86, 5687.

[20] Dundas, D., Taylor, K. T., Parker, J. S., and Smyth, E. S., 1999, J. Phys. B, 32, L231; Lappas, D. G., Corso, P. P., and Knight, P. L., 2000, Multiphoton Processes, edited by L. F. Diauro, R. R. Freeman and K. C. Kulander (Melville, NY: AIP), p. 245; Muller, H. G., ibid., p. 257; Panli, R., Szymanowski, C., Liu, W. C., and Eberly, J. H., ibid., p. 265.

[21] Chung, K. T., and Fullbright, P., 1992, Phys. Scripta, 45, 445.

[22] Lamb Jr., W. E., Schlicher, R. R., and Scully, M. O., 1987, Phys. Rev. A, 36, 2763.

[23] For recent reviews, see for example, Briggs, J. S., and Schmidt, V., 2000, J. Phys. B, 33, R1; King, G. C., and Avaldi, L., 2000, J. Phys. B, 33, R215.

[24] Maulbetsch, F., and Briggs, J. S., 1993, J. Phys. B: At. molec. opt. Phys., 26, 1679.

[25] McGuire, J. H., Berrah, N., Bartlett, R. J., Samson, J. A. R., Tanis, J. A., Cocke, C. L., and Schlachter, A. S., 1995, J. Phys. B, 28, 913.

[26] Teng, Z. J., and Shakeshaft, R., 1993, Phys. Rev. A, 47, R3487; 1994, ibid., 49, 3597. 